\title{
The use of covered stents in the field of interventional procedures for congenital heart defects
}

\author{
$3(2)=$ \\ Mario Carminati*, MD, FESC, FSCAI \\ Pediatric and Adult Congenital Cardiology Center, IRCCS Policlinico, San Donato, Milan, Italy
}

The use of covered stents in the field of interventional procedures for congenital heart defects has become progressively more and more popular in recent years, either as a bail-out after complications related to angioplasty or bare metal stent implantation, or as a first-choice procedure to prevent such complications or to treat some defects electively, such as fenestration closure as described in the paper by Butera et $\mathrm{al}^{1}$.

In this issue of EuroIntervention, Y. Boudjemline describes his experience with the use of a new covered stent (BeGraft; Bentley Innomed $\mathrm{GmbH}$, Hechingen, Germany), outlining the advantages as compared to other stents and, in particular, to the covered $\mathrm{CP}$ Stent $^{\circledR}$ (NuMED Inc., Hopkinton, NY, USA), which was in fact the most commonly used covered stent in congenital interventional cardiology for many years ${ }^{2}$.

\section{Article, see page 1008}

The enthusiasm of the author for the BeGraft stent is justified by the excellent results obtained; however, we have to remember that the covered CP Stent "did the job" with excellent results as well in hundreds of institutions all around the world for many years. We should be cautious of abandoning it in favour of new ones; more multicentre data and long-term follow-up are needed. In the recent past, a new stent (Advanta ${ }^{\mathrm{TM}}$; Maquet, Rastatt, Germany) seemed to be very promising but was quickly abandoned because of serious problems (infolding) that occurred in clinical practice, as correctly reported by the author in his paper. The characteristics of the BeGraft stent are in fact different and apparently safer and the stent performs better in our experience also. An example of BeGraft implantation in a case of "extreme" coarctation is shown in Figure 1.

On the other hand, the BeGraft stent is premounted on a proprietary single balloon; some operators may still prefer to use the $\mathrm{CP}$ Stent because it can be mounted on a BIB balloon and therefore the expansion of the inner balloon may allow (in some instances) a fine position adjustment before inflating the outer balloon for 


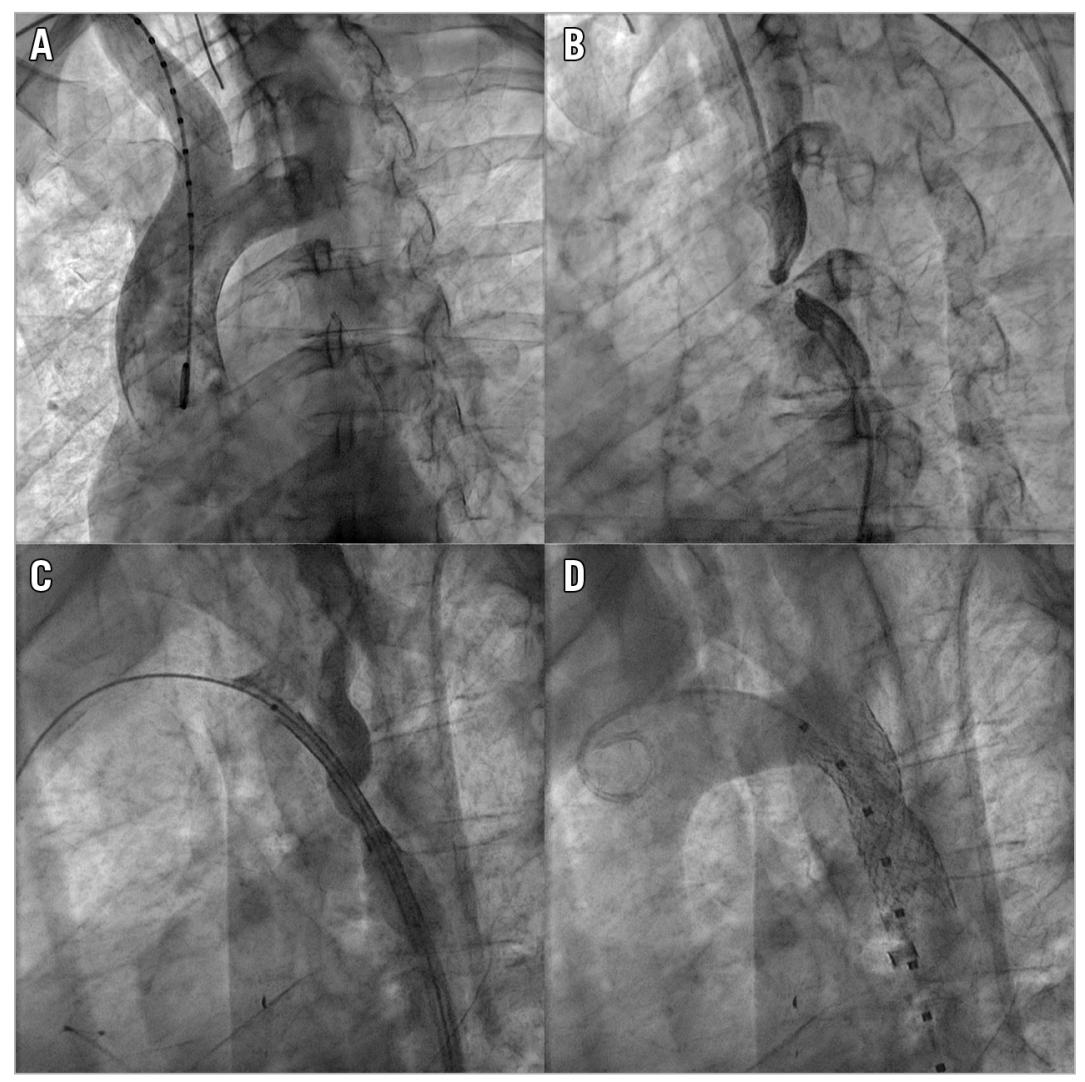

Figure 1. BeGraft implantation in a case of "extreme" coarctation. A) Aortogram in a case of "extreme coarctation" (or aortic atresia); the contrast medium opacifies the ascending aorta and transverse arch but cannot progress beyond the atretic isthmus. B) Simultaneous contrast injection above (through the radial artery access) and below (through the femoral artery access) the atretic segment. $C$ ) The atretic segment was perforated with the transseptal needle and a Mullins sheath was advanced from the femoral artery up to the transverse aortic arch. The BeGraft covered stent mounted on the balloon is seen within the sheath. D) Aortogram after BeGraft covered stent expansion. The stent is perfectly apposed to the vessel wall, restoring normal flow.

full stent expansion. Overall, the initial clinical experience with the BeGraft is very promising and it is likely to become more and more popular. To some extent the choice of material to be used is subjective and the final decision should be left in the hands of the individual operator.

\section{Conflict of interest statement}

The author has no conflicts of interest to declare.

\section{References}

1. Butera G, Manica JL, Marini D, Piazza L, Chessa M, Filho RI, Sarmento Leite RE, Carminati M. From bare to covered: 15-year single center experience and follow-up in transcatheter stent implantation for aortic coarctation. Catheter Cardiovasc Interv. 2014;83:953-63.

2. Boudjemline Y. Use of covered stents in the field of congenital heart diseases: the role of new players. EuroIntervention. 2018;14:e1008-10. 